\title{
Towards SDN/NFV-enabled satellite ground segment systems: Bandwidth on Demand Use Case
}

\author{
T. Ahmed ${ }^{1)}$, R. Ferrus ${ }^{2)}$, R. Fedrizzi ${ }^{3)}$, O. Sallent ${ }^{2)}$ \\ ${ }^{1)}$ CNRS-LaBRI, University of Bordeaux / Bordeaux INP, France; ${ }^{2)}$ Universitat Politècnica de Catalunya, Spain; ${ }^{3)}$ Center for \\ Research and Telecommunication Experimentation for Networked communities, Italy
}

\begin{abstract}
Key features of satellite communications such as wide-scale coverage, broadcast/multicast support and high availability, together with significant amounts of new satellite capacity coming online, anticipate new opportunities for satellite communications services as an integral part within upcoming 5G systems. To materialize these opportunities, satellite communications services have to be provisioned and operated in a more flexible, agile and cost-effective manner than done today. In this context, this paper describes the architecture of a satellite ground segment system that is built on the introduction of Software Defined Networking (SDN) and Network Function Virtualization (NFV) technologies and examines the use case for delivering a Satellite Bandwidth on Demand (BoD) solution.
\end{abstract}

Keywords-Satellite network; Network Function Virtualization; Software-Defined Networking; Satellite gateway virtualization; Combined satellite-terrestrial networks; service orchestration

\section{INTRODUCTION}

The role that satellite communications can play in the forthcoming $5 \mathrm{G}$ ecosystem is being revisited [1]. Technological advances in the satellite domain such as the use of High Throughput Satellites (HTS) in Geostationary Earth Orbit (GEO) [2] is changing the way that capacity is being brought to the market, reducing the price per bit and making it more attractive for other services such as satellite broadband communications. Likewise, a range of disruptive initiatives envisioning the use of non-GEO constellations with a large number of low-cost micro-satellites [3] might come to fruition in the forthcoming years, anticipating a further reduction in the cost of transmitting a bit over a satellite link while enhancing some of the Quality of Service (QoS) metrics such as latency.

In this context, the introduction of Software Defined Networking (SDN) and Network Function Virtualization (NFV) technologies within the satellite ground segment networks is anticipated to be a necessary step in their evolution [4][5]. SDN and NFV technologies can bring greater flexibility to Satellite Network Operators (SNOs), reducing both operational and capital expenses in deploying and managing SDN/NFVcompatible networking equipment as well as facilitating the integration and operation of combined satellite and terrestrial networks [6]-[8]. At the end of the day, SDN/NFV technologies are expected to better prepare SNOs for tomorrow's softwarised communications networks [9].
This paper describes an innovative architecture for SDN/NFV-enabled satellite ground segment systems and proposes a Satellite Bandwidth on Demand mechanism allowing to distribute bandwidth on-the-fly and on demand. The proposed architecture improves flexibility and reconfigurability in the delivery of satellite network services by supporting virtualization (i.e. softwarisation) of key satellite network functions together with network abstraction and resource control programmability. Moreover, the proposed architecture supports multi-tenancy to facilitate Virtual Network Operator (VNO) models and federation capabilities for the multi-domain orchestration of network functions and SDN-based control and management across terrestrial and satellite domains.

The rest of this paper is organized as follows. Section II outlines the main architectural traits of the proposed satellite ground segment system architecture. On this basis, section III describes SDN-based mechanisms to provide on-demand and dynamic bandwidth allocation between different Satellite Virtual Network Operators (SVNO). Furthermore, in section IV techniques for dynamic cross-layer QoS service will be presented to deal with capacity drop of the SVNO (e.g. due to bad weather conditions). Finally, conclusions are drawn in Section V.

\section{SDN/NFV-ENABLED SATELLITE GROUND SEGMENT SYSTEMS}

A Virtualized Satellite Network (VSN) is conceived here as a satellite network in which most of their functions are supplied as software components running in one or several Network Function Virtualization Infrastructure Point of Presence (NFVIPoPs). The non-virtualized functions of a VSN are provided through one or several Satellite Baseband Gateway-PNFs (SBGPNFs), which could be dedicated to a given VSN or shared among several VSNs. The operation of each VSN could be delegated to the customer/tenant, acting as a satellite VNO. Each VSN may be customized to the customer/tenant's needs, including a variety of different network services running as VNFs (e.g. PEP, VPN, etc.). In particular, as illustrated in Fig. 1, the following entities could form part of a given VSN: (1) one or several Satellite Network Function VNFs (SNF-VNFs) and SBG-VNFs that jointly provide the data plane functions of the VSN; (2) control applications and SDN controllers (all running as VNF instances) for the realization of some VSN control functions (e.g., QoS control, radio resource management 
[RRM], gateway diversity [GWD], Fading Mitigation Techniques [FMT], etc.); and (3) Network Management (NM) and Element Management (EM) functions, also running as VNFs, which provide a package of VSN management functions (e.g. Fault, Configuration, Accounting, Performance and Security [FCAPS] management). Another key feature of the architecture is the support for cross/multi-domain control and management capabilities through the exposition of control and management interfaces, as depicted in Fig. 1. Such interface will enable unified management when the VSN is interworked with terrestrial networks for e.g. end-to-end Traffic Engineering (TE). The virtualization of the satellite GW is studied in [10], identifying different variants with regard to which functions can be implemented as Virtualized Network Functions (VNF) and which remain as specialized hardware appliances (i.e. Physical Network Functions, PNFs). Without being conditioned to any particular variant, in the following we denote SNF-VNF to the implementation of the SNFs as VNFs, SBG-VNF to the implementation of part of the SBG functions as a VNF and SBGPNF to the non-virtualized part of the SBG functions.

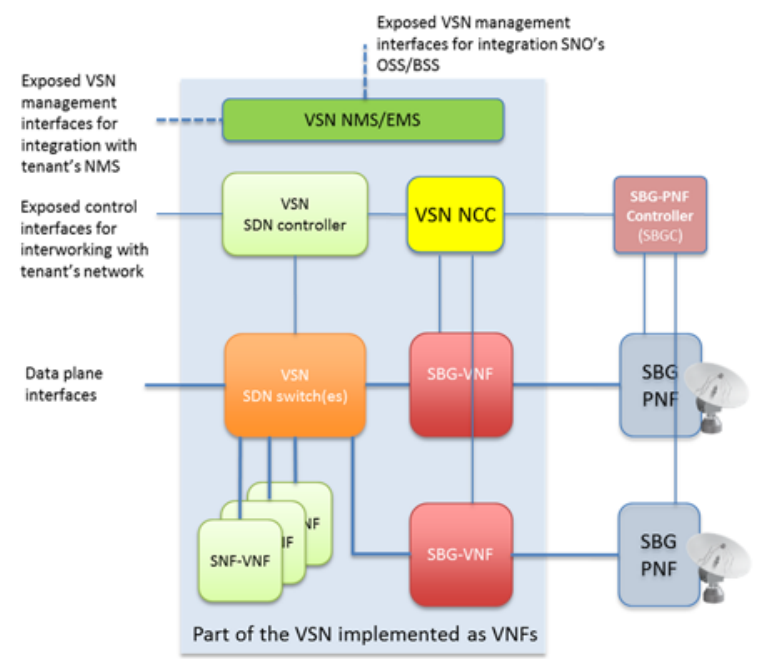

Fig. 1. Illustration of a multi-gateway VSN with integrated SDN controller that exposes control interfaces for interworking with the tenant's network

Together with the components previously discussed, the VSN depicted in Fig. 1 shows a multi-gateway satellite network with several SBG-VNF and SBG-PNFs along with multiple SNFVNFs. Without loss of generality, it is assumed that there is a single NCC and a single SBG-PNF Controller for the several gateways, even though other options would be possible. In addition, the VSN also comprises a number of SDN switches and a SDN controller which could be virtualized elements deployed as part of the VSN. The SDN switches are used to support the internal L3/L2 forwarding functions across the components of the VSN as well as for the interworking with external networks. As instance, SDN switches can add/drop VLAN tags/MPLS headers, enforce differentiate QoS treatments, etc., both for internal or for external interworking purposes. The VSN SDN controller is used (1) to control the operation of the SDN switches, (2) to support dynamic activation of the data transmission services (e.g. satellite bearer services [10]) through an interface with the NCC functions, and (3) to expose a control interface for interworking with the tenant's network. It's worth noting that Fig. 1 only intends to provide illustrative examples, introducing the main components of the VSN that are later on referred to in the description of the workflows. But other variants are possible depending on the types and characteristics of the VNFs that could be eventually conceived / developed to satisfy whatever specific needs (e.g., market demand, scalability issues, further split of functionality, etc.).

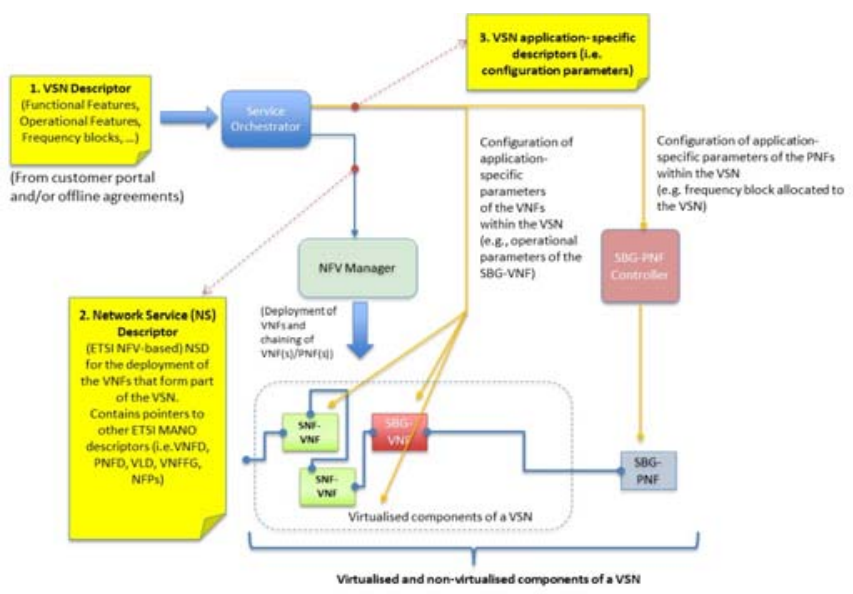

Fig. 2. Illustration of the VSN and the orchestration/management components.

To deploy and control the VSN, orchestration and management entities are needed as shown in Fig. 2. The network service orchestration capabilities are logically centralized in the socalled Service Orchestrator (SO) management component, which forms part of the Operation Support Systems / Business Support Systems (OSS/BSS) of a Satellite Network Operator (SNO). Beyond this, functionalities related to the instantiation, modification and termination of the VNFs that form part of the virtualised part of the VSN are covered by the NFV Manager. The functionalities provided by the SO and the NFV Manager are in charge of:

- Lifecycle management of the VSN, which can be defined as the set of functions required to manage the instantiation, maintenance and termination of a VSN.

- Composition of the network service descriptor (NSD) that represents the part of the VSN that is implemented as VNFs and executed over NFVI-PoP(s).

- Determination of the application-specific aspects of both VNFs and PNFs that form part of a VSN.

- Fault, Configuration, Accounting, Performance, Security (FCAPS) management of the VSN and its components, irrespective of whether they are VNFs or PNFs. These FCAPS management functionalities supported by the SO are mainly intended to guarantee the proper operation of the deployed VSN to the tenant/customer (e.g. performance measurement, resource usage, and alarms handling for accounting, reconfiguration and problem correction actions).

- Lifecycle management of the Network Service (NS) of a VSN through interaction with the NFV Manager, which offers the Os-Ma-nfvo reference point [13] as specified in the ETSI NFV MANO architecture (i.e. the SO is a consumer of reference point Os-Ma-nfvo). It's worth noting that the 
NFV Manager takes care of the deployment specific configuration of the VNFs that form part of the NS.

- Management of VNF packages that can be already onboarded on the NFV Manager or can be managed/onboarded onto it by the dashboard of the SO. In general, at the SO only the VNFDs are needed to compose the NSDs.

The operation of the SO and NFV Manager relies on a set of descriptors that are needed for the characterization of a VSN and its components. In general terms, a VSN Descriptor (VSND) is the input provided to the SO that describes the characteristics of the VSN as requested by the customer/tenant. Based on the VSND, the SO composes the NSD, which describes the virtualised part of the VSN, and the VSN application-specific descriptors, which contain the configuration of both VNFs and PNFs within the VSN. However, the details of the above mentioned components, extensively studied and developed in [4] are beyond the scope of this paper and introduced here for sake of clarity.

\section{SATELLITE BANDWIDTH ON DEMAND}

The main objective of the SDN-based flexible satellite Bandwidth-on-Demand (BoD) is to improve the typical satellite broadband access service with the ability to allow customer (SVNO, end-user) to dynamically resize (request and acquire) its bandwidth in a flexible manner. This concept is used to show how the granted SVNO bandwidth can be modified on-the-fly and on demand. In particular, in this section we illustrate the procedure to share the forwarding link bandwidth adapting it on the basis of the SVNOs requests. This implies some modifications in the VSN configuration. To study the concept of satellite bandwidth on demand, we suppose that the satellite forwarding link is a time division multiplexed broadcast link which is shared among two or many SVNOs. Each SVNO uses either a shared return link (shared TDMA) or a dedicated return channel (e.g. SCPC: Single Channel Per Carrier). The total carrier bandwidth of the forwarding link is supposed to be shared logically among the SVNOs. When the VSN of each SVNO is created, it will be assigned a nominal bandwidth described in its bandwidth profile according to the established SLA between the SVNO and the SNO (i.e. BW specification). The bandwidth profile contains different parameters such as Committed Information Rate (CIR) and Maximum Information Rate (MIR) that can be obtained during normal network operation (i.e. in clear sky condition using nominal MODCOD). Of course, the obtained throughput will depend on the actual MODCOD used by each remote which depends on the SNR level experienced by each terminal when using the Adaptive Coding and Modulation (ACM) mitigation technique. The SVNO bandwidth specification can be described as token bucket profile which can be subject to conformance test and bandwidth policy. In this case, the host SNO can reject / accept any extra bandwidth demand for any out-of-profile request according to the configured policies.

Realizing bandwidth on demand procedure will allow the SVNO to move from fixed bandwidth assignment to on-the-fly and on demand bandwidth assignment. Thus, we introduced more flexibility in assigning bandwidth based on the need of the
SVNO through an SDN-based flexible architecture to allow the total bandwidth available to be dispatched dynamically. Toward this, the total carrier bandwidth (owned by the SNO) is structured in a hierarchical way and divided into a bandwidth entities or bandwidth pool. Each SVNO is attributed an amount of bandwidth described as CIR and MIR which is the node in the hierarchy where the SVNO have an authority on. As such, the assigned bandwidth represents subdivisions of the total carrier bandwidth within that bandwidth pool. Each SVNO will further distribute its bandwidth for individual remotes and can allocate bandwidth to specific application using CIR/MIR specific to particular remote or group of remotes. Usually bandwidth granted to the SVNO is not shared with other SVNO as the resource should be separated to allow SVNO to manage properly its VSN and its QoS configuration. The SNO maintains an upto-date vision of the assigned bandwidth and total available bandwidth (amount of bandwidth requested, amount of bandwidth allocated, amount of free bandwidth).

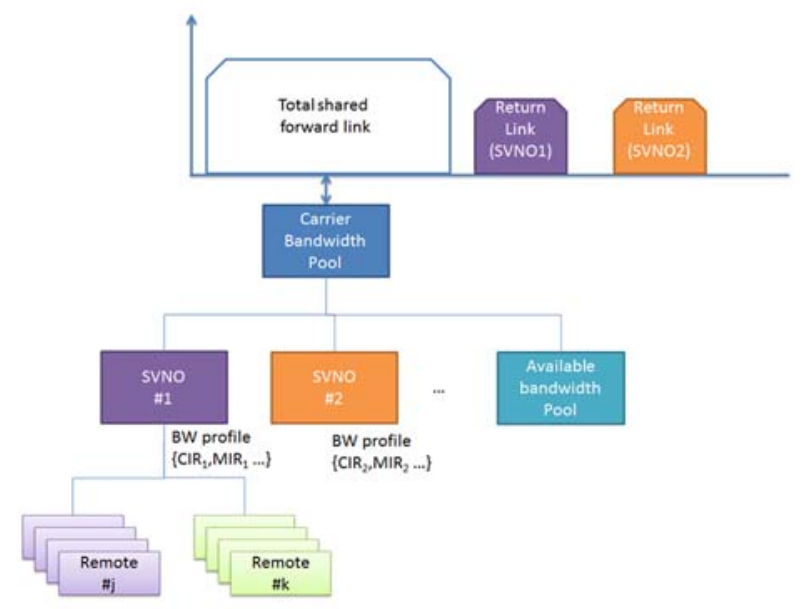

Fig. 3. Example of sample satellite network with two SVNO

Fig. 3 illustrates an example of a simple satellite configuration in which the total forwarding link capacity is granted to SVNO1 and SVNO2 according to their BW profiles. The available bandwidth can be distributed on request.

The attribution of bandwidth on demand on the bandwidth pool is only allowed by the SNO which exposes network service orchestration capabilities to the SVNOs through the SO. The SVNO must go through the SO decision to request scaling up or down its total bandwidth as shown in both scenarios of Fig. 4 and Fig. 5. Additional parameters related to this bandwidth can be requested by the SVNO such as quality of service parameters and service class. Several types of requests can be handled in the procedure of BoD:

- Simple request / response: there is no negotiation about the bandwidth profile that can be granted to the SVNO. BoD request can be accepted or refused by $\mathrm{SO}$ based on the available bandwidth pool.

- Complex request / response with negotiation: the procedure of granting BoD can use several round with multiple alternatives if possible, on which the SVNO can react. This can be coupled with QoS parameters in which the SVNO can 
select (accept/refuse) or make new request. This process can still until an agreement can be reach or a fail.

- Modification of bandwidth request parameters such modification in the parameter of the bandwidth profiles coupled with the parameter of the QoS class.

From a network architecture viewpoint, Fig. 4 and Fig. 5 show two possible approaches for sharing the capacity of the forwarding link between two SVNOs.

In Fig. 4, network (SBG-VNF and related control and management functions denoted here as Network Control CentreVNF [NCC-VNF] and Network Management Centre-VNF [NMC-VNF]) and spectrum are shared by the tenants (SVNO1, SVNO2) who provide the service to their end-users. This scenario is common in mobile network world in which the participation of the cost can be on basis of equality. In our case, the hosting SNO can operate and manage the network and spectrum and sells its capacity (bandwidth) to both tenants. The selling can be done through a broker who can play the role of intermediate or directly through an appropriate procedure. Once the network capacity is obtained, an SVNO can re-sell it to its customers. In BoD procedure scenario, the bandwidth sharing is done dynamically. A mixture between static and dynamic sharing can also be envisioned. In this case, the tenants have a minimum fixed capacity at their disposal and extending or decreasing this capacity would require invoking the BoD procedure dynamically.

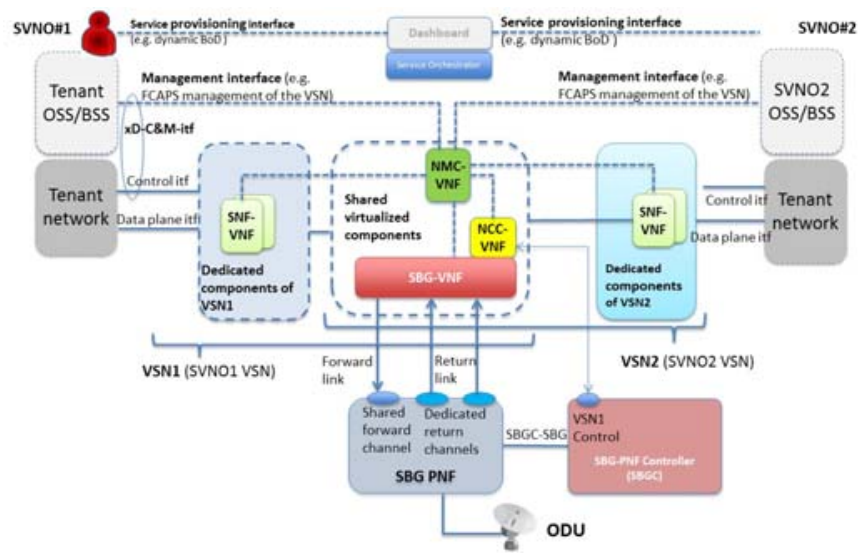

Fig. 4. Architectural view of two SVNOs' VSN sharing the same satellite forwarding link resources through common SBG-VNF and SBG-PNF

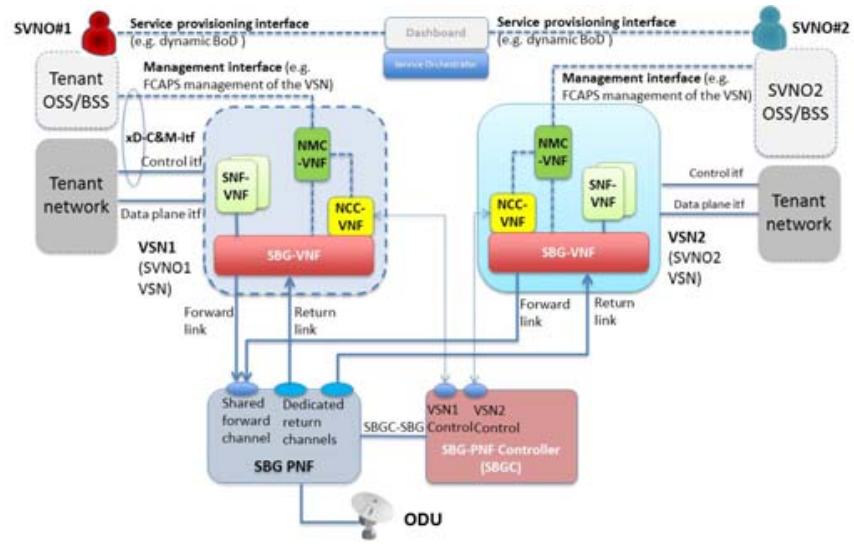

Fig. 5. Architectural view of two SVNOs' VSN sharing the same satellite forwarding link resources through dedicated SBG-VNF and common SBGPNF

In Fig. 5, the network is dedicated but the spectrum is shared on forward link and dedicated on the return link. Network resources are instantiated as a dedicated network slice with dedicated SNFVNF, SBG-VNF, NMC-VNF, NCC-VNF. The distribution of the shared resources could be done dynamically using BoD procedure. In this scenario the hosting SNO decide the amount of the network resources (BW) to distribute to each tenant based on actual network state. We could imagine an advanced scenario in which there is cooperation between tenants either directly or through a broker to perform real-time adjustment on their resources request in a form of a trading (buy/sell mechanisms) and grant a dynamic distribution of bandwidth access rights.

The invocation of the $\mathrm{BoD}$ procedure can be done through a service-provisioning interface to the SO allowing the SVNO is to request changes in the VSN bandwidth configuration. Interaction with the customer could be done through either a Dashboard/Customer Portal or a API built for this purpose. Another option could be the exposure of BoD capabilities through the control and management interfaces of the VSN itself (e.g. xD-C\&M-itf interface described in [11]) so that bandwidth adjustments could be done at some extent at VSN level without having to go necessarily through the SO. The former option of delivering the BoD service through a Dashboard/SO is the one further elaborated in the following and depicted in Fig. 6.

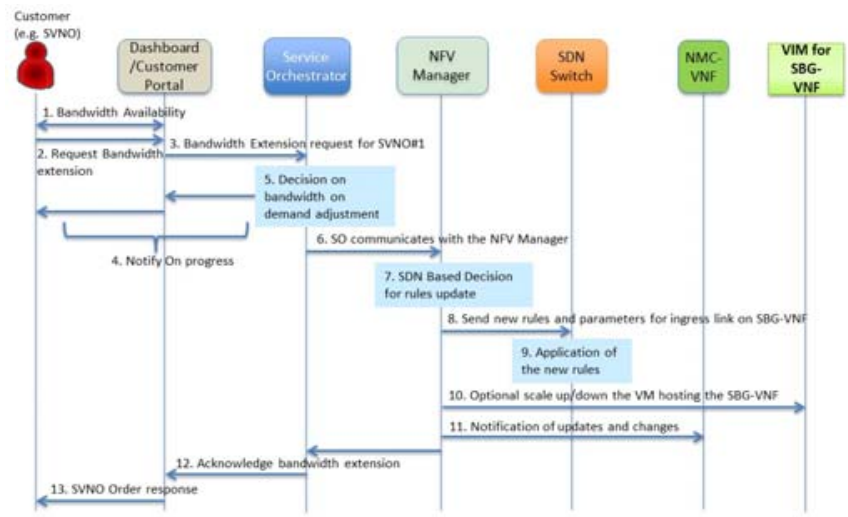

Fig. 6. Satellite Bandwidth-on-Demand for SVNO

Step 1: The customer (SVNO) is checking on his dashboard availability for bandwidth extension and possibility to request change in bandwidth profile granted to it.

Step 2: The customer sends a request for bandwidth on demand extension / reduction to scale up or down his network bandwidth.

Step 3: The request is forwarded to the service orchestrator who have the global view on the hosting network and who can decide to change the logical allocation of bandwidth based on currently attributed bandwidth among different SVNO and the available bandwidth.

Step 4: The customer is notified about the SO decision through his dashboard that changes are on progress.

Step 5: The service orchestrator takes the decision for deploying and configuring the new configuration for bandwidth 
extension and adjustment. In this case, it is assumed that changing the configuration rules of the SDN-enabled switch that links the SNF-VNFs and the SBG-PNF performs the bandwidth adjustment.

Step 6: The SO communicates with the NFV Manager to modify the configuration of the links.

Step 7: Driven by the NFV Manager, the corresponding SDN controller decides the new rules that should be applied for the SDN-enabled switch using the southbound communication protocols. This rules aims to make the egress traffic from the VSN (output stream of SGB-VNF) conformant to the bandwidth profile of the SVNO. In particular, the rule concerns the configuration of the token bucket mechanism that will be used for traffic conformance test. Among the configuration parameters that need to be configured, there are: the token burst size or CIR, the MIR and the measurement interval. Once this step is performed, out-of-profile and in-profile traffic has to be managed properly. For example, out-of-profile traffic can be discarded or accepted with less priority if there are available resources.

Step 8: the SDN controller send these rules to the switch.

Step 9: the SDN-enabled switch applies the received rules.

Step 10: To properly handle the bandwidth adjustment, the NFV manager can optionally scale (up / down) the VM hosting the SBG-VNF.

Step 11: The NFV Manager notifies the NMC-VNF to consider these changes to take into consideration the new bandwidth profiles to be used on the VSN.

Step 12 and 13: The NFV-Manager notifies the SO, which notifies the customer using dashboard about the BoD adjustment.

The above procedure basically covers the case that the bandwidth adjustment does not require any modification of the SBG-PNF settings. In case that a reconfiguration of the resources allocated in the SBG-PNF was needed, SO should communicate with both the NFV Manager and the SBG-PNF Controller to update the VSN configuration.

To provide the above-mentioned procedure, there should be multiple functional blocks implemented inside the SO and the NFV Manager that allow providing BoD such as:

- BoD request handling: the role of this entity is to handle the request between the SVNO and the satellite network and to response to the SVNO. The outcome of the BoD request handling process can also trigger the reconfiguration of the related bandwidth in relationship with the NFV manager.

- BoD request policies: this block allows influencing on the acceptance of the BoD request, for example to check if the SVNO is eligible to request.

- Bandwidth pool availability database.

\section{CROSS-LAYER OPERATION TOGETHER With SATELLITE} MAC SCHEDULERS

Bandwidth is not the only parameter that SNO / SVNO require for its normal operation. Many applications are very sensitive to delay, jitter and error rates such as VoIP, network games, transactions, and streaming applications. Being able to implement cross-layer bandwidth on satellite network is considered as a necessity to meet SVNOs and customers' expectations to deliver a QoS-enabled service. The delivery of such service should take into consideration the actual network condition and would require advance mechanisms that enable efficient traffic filtering, classification, marking and advance control on the outgoing and ingoing traffic on the satellite gateway. The traffic matching a particular profile can be assigned an appropriate traffic class (TC) to meet the service requirements. The implementation of such $\mathrm{TC}$ can be done using different types of queues both at IP and MAC levels as illustrated in Fig. 7.

Each queue can have different weight and priority depending on its configuration. Finally, the queues are attached a mechanism for traffic scheduling. The configuration parameters and setting of all these components can be controlled dynamically and not statically as it is common. Thus, the crosslayer QoS service can be seen as an SDN-enabled application that facilitate dynamic provisioning of configuration parameters and setting of QoS building blocks (filter, classifier, marker, admission control, policies, shaper, queue parameters, scheduler...). In particular, depending on the current network and weather conditions, this application allows determining, how packets are filtered, scheduled and prioritized as they flow through the network. SDN decision is used to make the decision on how to filter and to classify this traffic and to mark it to be later on correctly scheduled. It also takes a decision on how to perform rate limiting, quotas and shaping policies. All these decisions are based on cross-layer information that takes into account L7 to L1 information and not only information on particular layer.

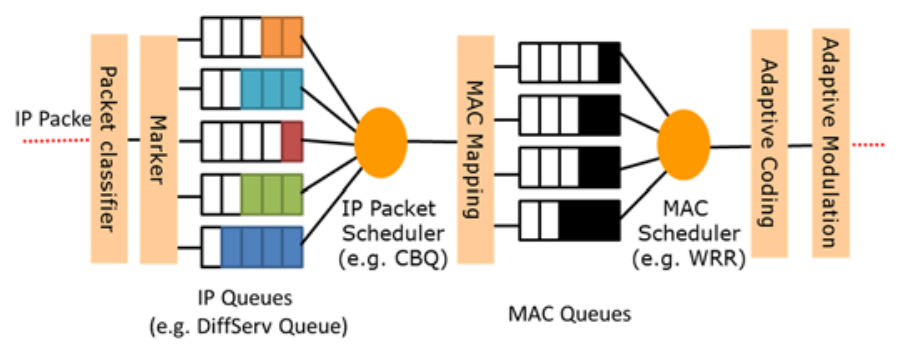

Fig. 7. Basic scheduler model

We illustrate in the following an example of cross-layer QoS mechanisms used to configure the packet scheduling parameters on a particular queue to deal with the problem of capacity drop under bad weather conditions. We concentrate only on scheduler between the network and PHY layer. In the DVB-S2 standard, the scheduling discipline is assumed to be Weighted Round Robin (WRR) that serves different queues. Each queue is served with a particular serving weight (Wi). The objective of cross-layer QoS is to keep the transmission delay on the forwarding link (delay from the gateway to a satellite terminal) under a certain limit for the high priority queue. Thus, when a terminal is affected by some bad weather conditions (rain/clouds), the transmitted information on the physical layer has to be increased to be able to send the same useful data as 
before. This leads to useful capacity drop and length increase in this queue. To mitigate this problem, it is possible to change dynamically the serving weight of this queue to be increased to serve more data. This leads to Adaptive WRR schema in which the different queue weights are calculated dynamically as illustrated in Fig. 8. This cross-layer schema has been used in the past in many works to provide adaptive packet scheduling to support QoS [14] or with QoS and fairness [15][16] over satellite systems. The novelty in this work is the application of SDN-based cross-layer QoS and providing automatic weights configuration with accurate and suitable value to meet the service constraints. This weight calculation can take into consideration the current network capacity along with the MODCOD used, SNR level, and queue size.

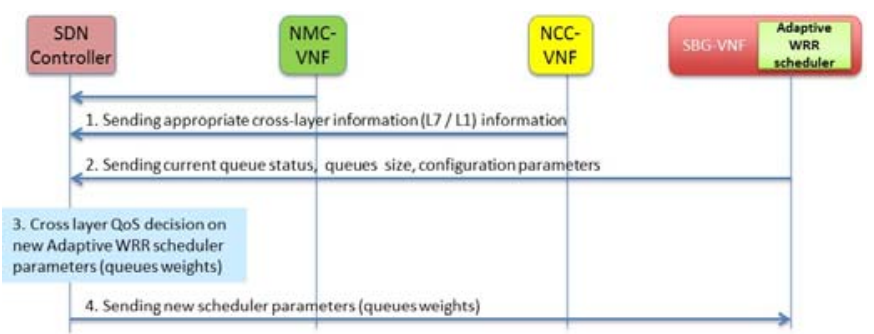

Fig. 8. Cross-Layer QoS procedure for Adaptive WRR scheduler

The steps involved in the cross-layer QoS procedure are described bellows:

Step 1 and 2: The SDN-based cross-layer QoS application within the SDN-controller receives the appropriate monitoring information needed to take the decision.

Step 3 and 4: The SDN controller decides on the new scheduler parameters and sends the corresponding rules.

\section{CONCLUSIONS}

The adoption of SDN and NFV technologies into the satellite domain is one of the key facilitator to enhance the delivery of satellite communications services and achieve a better integration of the satellite component within the $5 \mathrm{G}$ ecosystem. This paper has described an architecture of a SDN/NFV-enabled satellite ground segment system that supports on-demand dynamic orchestration of satellite communications services. Together with the main architectural traits, procedures for the realization of diverse SDN-based control and management solutions within satellite ground segment systems have been elaborated. In particular, a procedure for Bandwidth on Demand (BoD) has been presented which allows dynamic allocation and sharing of resources between different SNO tenants. Moreover, a procedure for dynamic cross-layer QoS service have been specified allowing the dynamic configuration of scheduling parameters to deal with capacity drop of the SVNO, due to bad weather conditions. The main novelty of the proposed work is the application of SDN principles in the virtualized satellite ground segment to provide firstly mechanisms for sharing resources between SNO tenants and, secondly, provide dynamic scheduling parameters configuration to meet SVNOs expectations to deliver QoS-enabled services to their customers.

\section{ACKNOWLEDGMENT}

Research leading to these results has received funding from the European Union's H2020 Research and Innovation Programme (H2020-ICT-2014-1) under the Grant Agreement H2020-ICT-644843.

\section{REFERENCES}

[1] Marius Corici, Adam Kapovits, Stefan Covaci, Alexander Geurtz, IlieDaniel Gheorghe-Pop, Björn Riemer, Andreas Weber. "Assessing satellite-terrestrial integration opportunities in the $5 \mathrm{G}$ environment". [Online] Available: https://artes.esa.int/sites/default/files/Whitepaper\%20\%20Satellite_5G\%20final.pdf [Feb. 27, 2017]

[2] Fenech, H.; Amos, S.; Tomatis, A.; Soumpholphakdy, V., "High throughput satellite systems: An analytical approach," IEEE Transactions on in Aerospace and Electronic Systems, January 2015

[3] ARTES programme, "ESA announces dedicated support for the development of megaconstellations", Last updated July 2015. Available online at https://artes.esa.int/news/esa-announces-dedicated-supportdevelopment-megaconstellations

[4] H2020 VITAL research project website at http://www.ict-vital.eu/

[5] Bertaux L., Medjiah S., Berthou P., Abdellatif S., Hakiri A., Gelard P., "Software Defined Networking and Virtualization for Broadband Satellite Networks". IEEE Communications Magazine, March 2015

[6] Sacchi, C.; Bhasin, K.; Kadowaki, N.; Vong, F., "Toward the "space 2.0" Era [Guest Editorial]," Communications Magazine, IEEE , vol.53, no.3, pp.16,17, March 2015

[7] NetWorld2020's - SatCom WG The role of satellites in 5G, Version 5 31 th July 2014

[8] 3GPP TR 22.891 V1.1.0, "Feasibility Study on New Services and Markets Technology Enablers;Stage 1 (Release 14)", November 2015

[9] R. Ferrus, H. Koumaras, O. Sallent, G. Agapiou, T. Rasheed, M.-A. Kourtis, C. Boustie, P. Gelard, T. Ahmed, SDN/NFV-enabled satellite communications networks: Opportunities, scenarios and challenges, Physical Communication, November 2015

[10] Toufik Ahmed, Emmanuel Dubois, Jean-Baptiste Dupé, Ramon Ferrús, Patrick Gélard and Nicolas Kuhn, "Software Defined Satellite Cloud RAN". Under submission.

[11] H2020 VITAL Deliverable D2.3, http://www.ictvital.eu/documents/deliverables

[12] NetWorld2020, "Public Private Partnership in Horizon 2020: Creating a Smart Ubiquitous Network for the Future Internet", November 2013

[13] ETSI GS NFV-MAN 001 V1.1.1 (2014-12), "Network Function Virtualisation (NFV); Management and Orchestration"

[14] Rendon-Morales, E., Mata-Díaz, J., Alins, J., Muñoz, J. L., \& Esparza, O. (2011). Adaptive packet scheduling for the support of QoS over DVB-S2 satellite systems. In Wired/Wireless Internet Communications (pp. 1526). Springer Berlin Heidelberg.

[15] Castro, M., \& Granados, G. S. (2007). Cross-layer packet scheduler design of a multibeam broadband satellite system with adaptive coding and modulation. Wireless Communications, IEEE Transactions on, 6(1), 248-258.

[16] Vieira, F., Castro, V., \& Seco Granados, G. (2006). A tunable-fairness crosslayer scheduler for DVB-S2. International Journal of Satellite Communications and Networking, 24(5), 437-450 